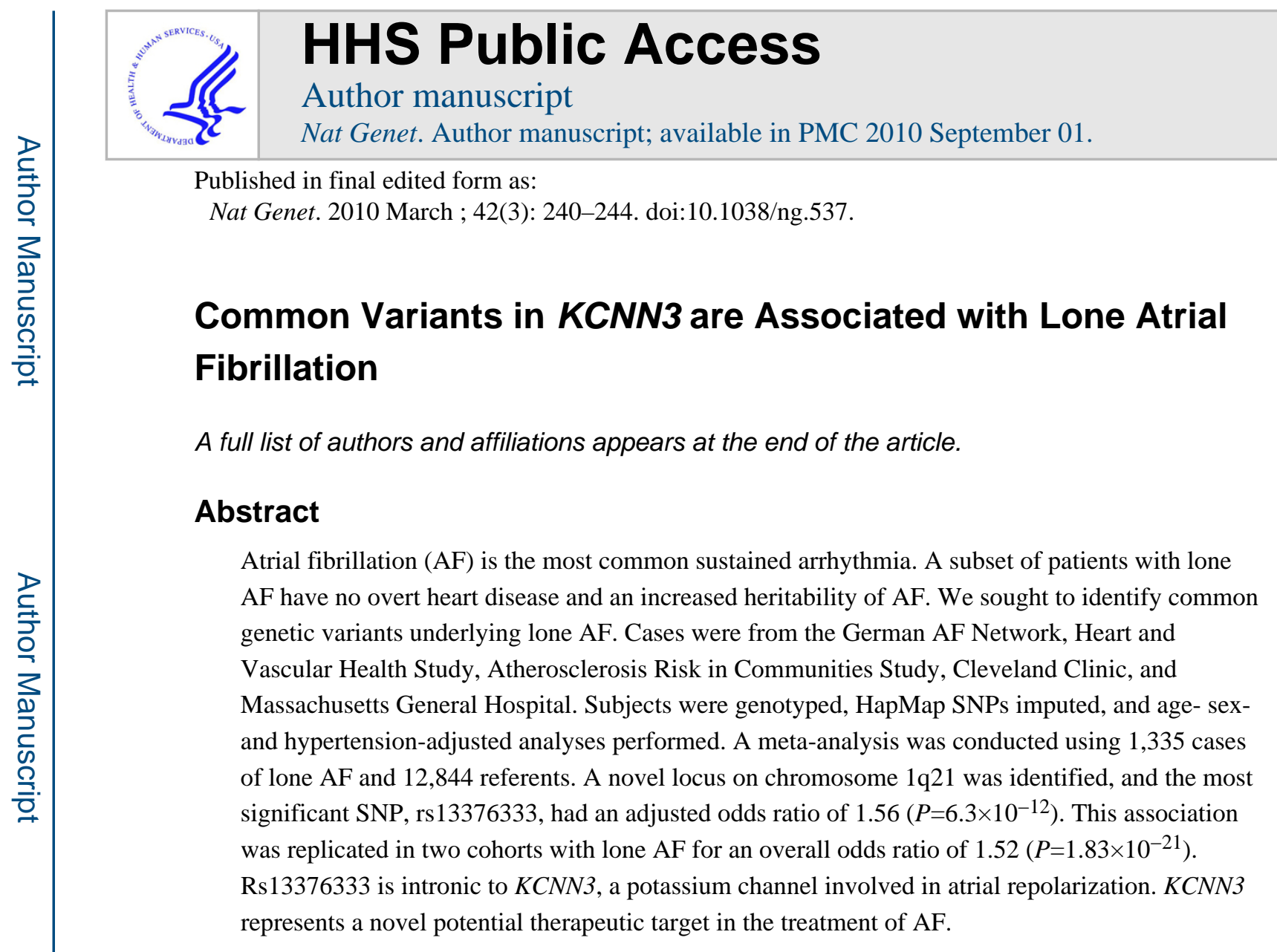

\begin{abstract}
AF results in significant morbidity and mortality. Risk factors for typical AF include advanced age, male sex, obesity, hypertension, heart failure, valvular heart disease,
\end{abstract}

\footnotetext{
Users may view, print, copy, download and text and data- mine the content in such documents, for the purposes of academic research, subject always to the full Conditions of use: http://www.nature.com/authors/editorial_policies/license.html\#terms

Corresponding Authors:Patrick T. Ellinor, MD, PhD, Cardiac Arrhythmia Service and Cardiovascular Research Center, Massachusetts General Hospital, Boston, MA; Phone 617-724-8729, FAX: 617-726-5806, pellinor@ partners.org. Stefan Kääb, MD, PhD, LMU München, Klinikum Großhadern, Department of Medicine I, 81366 München, Germany; Phone: +49-89-70-95-6049, FAX: +49-89-7095-6076 Stefan.Kaab@med.uni-muenchen.de..

60 These authors contributed equally to the manuscript.

Current address: Cardiology Center, Geneva University Hospital, Geneva, Switzerland

Author Contributions Study concept and design: P.T.E, K.L.L., N.L.G., A.P., M.K.C., A.A., J.C.M.W., D.E.A., E.J.B., S.R.H., S.K. Acquisition of data: P.T.E, K.L.L., N.L.G., A.P., A.A., M.K.C., M.F.S., P.I.W.B., M.M., S.A.L., E.F., D.D., N.L.S., J.D.S., R.B.S., E.Z.S., K.M.R., D.R.V.W., B.-M. B, C.N., K.W., G.B.E., J.I.R., S.L.H., G.S., A.V.S., L.J.L., T.B.H., S.M., M.Ne., D.J.M., S.P., T.E., S.M., C.N.C., M.L., S.M., K.W., T.J.W., L.K., E.B., V.G., B.M.P., J.C.M.W., J.B., D.E.A., E.J.B., S.R.H., S.K. Analysis and interpretations of data: K.L.L., N.L.G., A.P, M.M, J.B., D.E.A., K.W.

Drafting of the manuscript: P.T.E, K.L.L., E.J.B., S.R.H., S.K.

Critical revision of the manuscript for important intellectual content: K.L.L., N.L.G., A.P., A.A., M.K.C., M.F.S., P.I.W.B., M.M., S.A.L., E.F., D.D., N.L.S., J.D.S., R.B.S., E.Z.S., K.M.R., D.R.V.W., B.-M.B., C.N., K.W., G.B.E., E.Z.S., S.L.H., G.S., A.V.S., L.J.L., T.B.H., S.M., M.Nö., D.J.M., S.P., T.E., A.K., S.M., C.N.C., M.L., S.M.,T.J.W., L.K., R.S.V., M.Ne., C.A.M., B.H.Ch.S., A.H., A.G.U., D.L., E.B., A.M., E.J.T., A.C., V.G., B.M.P., D.M.R., T.M., H.-E. W., J.C.M.W., J.B., D.E.A., E.J.B., S.R.H. Statistical analysis: K.L.L., N.L.G., A.P., M.M., J.B., D.E.A., K.W.

Obtained funding: P.T.E, A.P., A.A., M.K.C., M.F.S., P.I.W.B., M.M., S.A.L., E.F., N.L.S., J.D.S., K.M.R., D.R.V.W., J.I.R., S.L.H., S.M., B.H. Ch. S., A.H., A.G.U., D.L., E.B., A.M., E.J.T., A.C., V.G., B.M.P., D.R., T.M., J.C.M.W., J.C.M.J., J.B., D.E.A., E.J.B., S.R.H., S.K.

Study supervision: P.T.E, K.L.L., N.L.G., A.P., A.A., M.K.C., M.F.S., J.C.M.W., J.B., D.E.A., E.J.B., S.R.H., S.K..

Had full access to all of the data in the study and takes responsibility for the integrity of the data and the accuracy of the data analysis: P.T.E, K.L.L., N.L.G., A.P., A.A., M.K.C., M.F.S., J.C.M.W., J.B., D.E.A., E.J.B., S.R.H., S.K.
}

Financial Disclosures: Dr. Aravinda Chakravarti is a paid member of the Scientific Advisory Board of Affymetrix, a role that is managed by the Committee on Conflict of Interest of the Johns Hopkins University School of Medicine. 
hyperthyroidism and family history of AF. A subset of patients with AF exhibit no clear precipitant, have no structural heart disease, present at a young age, and are referred to as having lone AF.1 Patients with lone AF are more likely to have symptomatic, paroxysmal episodes than older individuals with more typical forms of AF observed in the community. In many patients with lone $\mathrm{AF}$, discrete foci of ectopic electrical activity that initiate $\mathrm{AF}$ have been found to originate within the pulmonary veins. Although lone AF appears to have a more benign course than typical AF, 2 many symptomatic patients require treatment with an antiarrhythmic medication, electrical cardioversion, catheter ablation procedure to electrically isolate the pulmonary veins, or a combination of therapies.

Familial aggregation and an early-onset is prominent in subjects with lone AF, and nearly $30 \%$ of probands with lone AF have a first-degree relative with the disease. 3 Framingham Heart Study (FHS) investigators observed that the odds of developing AF were 3 times higher for individuals with at least one parent in whom AF was diagnosed before age 75 years than in those without a parental history of AF.4 Similarly, in a large study of Icelanders, the risk of developing $\mathrm{AF}$ was increased nearly five-fold if one parent was affected before age 60 years. 5

A genome-wide association study (GWAS) in Icelanders identified a chromosome 4q25 locus associated with AF.6 Within this locus, two non-coding single nucleotide polymorphisms (SNPs) were independently associated with AF. The SNP most strongly associated with AF, rs2200733, conferred a 1.71 fold increased odds of AF $\left(\mathrm{p}=6.1 \times 10^{-41}\right)$, while rs 10033464 had a 1.42 fold increased odds of AF $\left(\mathrm{p}=3.1 \times 10^{-11}\right) .6$ Recently, a novel genetic locus for typical AF observed in the community was described.7,8 SNP rs2106261 on chromosome 16q22 was associated with AF with a risk ratio of $1.25\left(p=1.8 \times 10^{-15}\right) .7$

Using well-defined and carefully phenotyped subsets of patients with a disease can facilitate gene discovery for complex traits such as AF. Lone AF remains a diagnosis of exclusion and a consensus definition has yet to emerge.9 In the current study, lone AF was defined as AF with an onset prior to 66 years of age and without a preceding history of a myocardial infarction, heart failure, or known left ventricular systolic dysfunction. Since subjects with lone AF have a high heritability of AF and a distinct clinical presentation, we sought to identify the common genetic factors underlying lone AF.

A total of 1,335 subjects with lone $\mathrm{AF}$ and 12,844 referent subjects with genome-wide genotyping were available from five centers (Table 1). After application of the SNP exclusion criteria in each study (Supplemental Table 1) the genomic inflation factor $(\lambda)$ ranged from 0.98 in ARIC to 1.17 in $\mathrm{HVH} / \mathrm{CHS}$, and the overall genome-wide meta-analysis $\lambda$ was 1.017. Supplemental Figure 1A displays the quantile-quantile plot of the expected versus observed $P$ value distributions for associations of the approximately 2.5 million SNPs with lone AF. Figure 1 illustrates the results of the lone AF meta-analysis with the $-\log _{10}(P$ value) plotted against physical coordinates on 22 autosomal chromosomes. The most significant association with lone AF was at the previously reported 4q25 locus with 77 SNPs that exceeded the pre-specified genome-wide significance $P=5 \times 10^{-8}$. The most significant SNP at this locus was rs6843082 (OR 2.03, 95\% CI, 1.79-2.30; $P=2.5 \times 10^{-28}$, Table 2, Figures 1, Supplemental Figure 2A, and Supplemental Table 2). Given the large number of 
significant associations at this locus, a modified quantile-quantile plot without the 4q25 SNPS is presented in Supplemental Figure 1B.

A second locus identified on chromosome 1q21 had 6 SNPs that exceeded genome-wide significance. The most significant SNP was rs13376333 with an OR of 1.56 (95\%CI 1.38$1.77, P=6.3 \times 10^{-12}$, Table 2, Figures 1 and 2, Supplemental Table 2). SNP rs13376333 is located in the intron between the first and second exon of the calcium activated potassium channel KCNN3 (NM 002249). A regional plot of the locus on chromosome 1q21 is illustrated in Figure 2. Our findings at the chromosome 1q21 locus were replicated in two studies with lone AF. First, in 977 cases from the AFNET and 3,042 controls without AF from KORA S4, rs13376333 was significantly associated with lone AF (OR 1.45,95\% CI $\left.1.26-1.66, \mathrm{p}=8.8 \times 10^{-8}\right)$. Second, the association was replicated in the Vanderbilt University Lone AF Registry consisting of 187 subjects with lone AF and 565 control subjects without $\mathrm{AF}$ (OR $1.5595 \% \mathrm{CI} 1.19-2.03, \mathrm{p}=0.001$ ). In a meta-analysis combining the lone $\mathrm{AF}$ results of the primary GWAS and the two replication cohorts, rs13376333 had an OR of 1.52 $\left(95 \%\right.$ CI $\left.1.40-1.64, \mathrm{p}=1.83 \times 10^{-21}\right)$.

There was no evidence of an interaction between SNPs rs6843082 and rs13376333, the most significant SNPs at the chromosome $4 \mathrm{q} 25$ and 1q21 loci, respectively $(P=0.46)$. We also sought to determine if rs 13376333 was associated with more typical forms of AF observed in the community. Using GWAS data from two independent studies in Rotterdam and Reykjavik (Age, Gene/Environment Susceptibility Study or AGES), we found that SNP rs13376333 was associated with AF, albeit with a weaker effect (Supplemental Table 3).7 In a meta-analysis of the associations between this SNP and both incident and prevalent AF in Rotterdam and AGES, rs13376333 had an OR of 1.13, (95\%CI 1.04-1.24, $P=0.006$ ).

A third SNP, rs13038095 on chromosome 20q13, also exceeded genome-wide significance (OR 1.61, 95\% CI 1.37-1.91, $\mathrm{p}=1.1 \times 10^{-8}$, Table 2 and Supplemental Table 2 ) and is located upstream from the endosulfatase, SULF2 (Supplemental Figure 2B). However, in the AFNET and Vanderbilt studies, the association with AF was in the opposite direction and thus failed to replicate (Table 3). Finally, we confirmed the recent locus for AF on chromosome 16q22 described in a meta-analysis of GWAS data from five longitudinal cohorts with more typical forms of AF observed in the community. The minor allele of rs2106261 was associated with lone AF with an odds ratio of 1.47 (95\% CI 1.39-1.54, $\left.\mathrm{p}=1.61 \times 10^{-7}\right)$.

Variants at the 1q21 locus cluster at the $K C N N 3$ gene which codes for a member of a family of voltage-independent calcium-activated potassium channels (also known as $S K 3$ or $K C a 2.3)$. These small conductance channels are expressed in a number of excitable tissues including the brain10 and heart.11,12 In neurons, these channels underlie the classical afterhyperpolarization or inward potassium current that is activated by elevations in calcium following repetitive action potential stimulation. The role of these channels in the heart is less clear. In a rabbit burst pacing model designed to simulate pulmonary venous ectopy, pharmacologic blockade of KCNN channels inhibited pacing induced shortening of pulmonary venous and atrial action potential duration.13 Shortening of the atrial action potential duration reduces the refractory period of atrial myocytes and promotes reentry, an 
important mechanism for the development and maintenance of AF.14 While no change was observed in $K C N N 3$ expression in response to burst pacing, 13 expression of a closely related family member, $K C N N 2$, was increased. Differential expression of KCNN2 in a mouse model is associated with altered pacemaker cell action potential duration and spontaneous firing rate. $15 \mathrm{KCNN} 3$ and $\mathrm{KCNN} 2$ co-assemble in heteromultimeric channel complexes in vitro, 16 therefore genetic variation in either subunit may modulate channel function. However, we did not identify any SNPs within 60 kilobases of $K C N N 2$ that were significantly associated with lone AF (defined as $P<0.001$, data not shown). The KCNN channels also are expressed in vascular endothelial cells, and suppression of $K C N N 3$ expression in a mouse model has been associated with increased blood pressure.17 The most significant SNP at this locus, rs13376333, is not in LD with any known common, nonsynonymous SNP in $K C N N 3.18$ Thus, the mechanism by which variation at this locus increases AF risk is unknown. While $K C N N 3$ is a plausible candidate gene, additional studies on the role of this channel and other genes at this locus are warranted. Future work directed at determining the role of the identified SNPs for lone AF in predicting the progression from paroxysmal to permanent AF or in defining subtypes of lone AF will be of interest. Furthermore, the utility of $K C N N 3$ as a potential direct or indirect target for the pharmacological treatment of AF may also merit further investigation.

To minimize false positive associations that may arise from GWAS, we used a conservative threshold for genome-wide significance and independently replicated our findings in additional subjects with lone AF. To avoid the confounding influence of population stratification, our analyses were restricted to subjects of European ancestry, so the generalizability of our findings to individuals of non-European ancestry may be limited. Additionally, we included subjects with hypertension in our analysis owing to the high lifetime risk of hypertension and to maximize the power of our study. However, it is unlikely that hypertension is confounding our results since SNP rs13376333 was not associated with systolic or diastolic blood pressure in the Global BPgen Consortium, a metaanalysis of genome-wide association data for blood pressure in over 34,000 subjects from 13 cohorts of European descent (systolic blood pressure $+0.2 \mathrm{~mm} \mathrm{Hg} /$ minor allele, $95 \% \mathrm{CI}-0.1$ to $+0.5, \mathrm{p}=0.27$ and diastolic blood pressure $+0.1 \mathrm{~mm} \mathrm{Hg} / \mathrm{minor}$ allele, $95 \% \mathrm{CI}-0.1$ to $+0.3, \mathrm{p}=0.25) .19$ Furthermore our results were replicated in subjects from Vanderbilt with lone AF in the absence of hypertension. Although we were not able to perform a multivariable analysis adjusting for other associated risk factors for AF, rs1337633 was not associated with BMI or left atrial size, two other common risk factors for AF (data not shown). Since lone AF is a diagnosis of exclusion, there were variations in the enrollment procedures between sites. In particular, an echocardiogram was not performed on subjects within the ARIC study nor approximately $30 \%$ of the subjects in the HVH study. Nevertheless, our results remained robust and were consistently replicated in independent populations with lone AF. Finally, the associations we observed do not necessarily imply that the candidate genes discussed are directly involved in the pathogenesis of AF. The associated SNPs may lie within or serve as proxies for non-coding regulatory elements that may affect gene expression at considerable distances from their respective genomic locations. 
In summary, we identified a novel locus for lone $\mathrm{AF}$ at the calcium-activated potassium channel gene $K C N N 3$. Future studies will seek to determine the mechanistic links between genetic variation at this locus and AF.

\section{Online Methods}

Lone AF was defined as AF with an onset prior to 66 years of age and without a preceding history of a myocardial infarction, heart failure, or known left ventricular systolic dysfunction. Referent subjects in all studies were free from AF as documented by electrocardiography. In each center the local institutional review boards reviewed and approved all study procedures; written, informed consent was obtained from each study subject. All subjects were of European descent. None of the cases or controls are related. None of the GWAS data in the cases with lone AF from MGH, AFNET, HVH, or CC have previously been reported. Cases with lone AF from the ARIC study were included in a metaanalysis of the genetic determinants of AF observed in the community.7

\section{Detailed Description of Study Cohorts}

The German Competence Network for Atrial Fibrillation (AFNET) 21 is a national registry of AF patients. In this registry, DNA samples have been collected from patients with AF onset before age 60 years at the Medical Department I of the University Hospital Munich, Campus Grosshadern of the Ludwig-Maximilians University Munich. Cases were selected if the diagnosis of AF was made on an electrocardiogram analyzed by a trained physician. Patients with signs of moderate to severe heart failure, moderate to severe valve disease or with hyperthyroidism were excluded from the study. Referent subjects were drawn from the KORA S4 study,22 with ages ranging from 25-74 years, and had no history of AF, myocardial infarction, heart failure or valve disease and documented sinus rhythm at the time of blood draw.

The Massachusetts General Hospital Atrial Fibrillation Study (MGH)3 enrolled serial patients with lone $\mathrm{AF}$ or $\mathrm{AF}$ and hypertension referred to the arrhythmia service between July 5, 2001 and February 19, 2008. Inclusion criteria were AF documented by electrocardiography, and age less than 66 years. Individuals with structural heart disease as assessed by echocardiography, hyperthyroidism, myocardial infarction, or heart failure were excluded. All patients were evaluated by 12-lead electrocardiogram, echocardiogram, and laboratory studies. Referent subjects were selected from the Framingham Heart Study (FHS),23-25 and included unrelated subjects 18-74 years of age and no history of AF at blood draw or in follow up, and no history of myocardial infarction, heart failure or valve disease at baseline.

The Heart and Vascular Health Atrial Fibrillation Study (HVH)26 identified all enrollees of Group Health, a large health plan in Washington State, with a new International Classification of Diseases, $9^{\text {th }}$ revision (ICD-9) code for AF between October 1, 2001 and December 31, 2004. Incident AF was verified by review of medical records with the requirement that $\mathrm{AF}$ be documented by 12-lead electrocardiogram and clinically recognized by a physician, with no previous evidence of AF in the medical record. Patients with early onset $\mathrm{AF}$ were those less than 66 years of age at diagnosis, without a history of coronary 
artery disease, valvular disease, heart failure, poor left ventricular function, chronic obstructive pulmonary disease, active cancer, or hyperthyroidism. Referent subjects were identified from the Cardiovascular Health Study (CHS), 27 and were aged 65-74 years, had no history of AF at blood draw or in follow up and no history of myocardial infarction, heart failure or valve disease at baseline.

Cases of early onset AF were identified from the Atherosclerosis Risk in Communities (ARIC) Study.28 Study participants underwent electrocardiograms at baseline and at each follow-up exam ( 3 exams; one exam every 3 years). As part of standard follow-up procedures, trained abstractors obtained and recorded all ICD-9 hospital discharge diagnosis from participant's hospitalizations reported in annual follow-up interviews. AF was defined as presence of ICD-9 code 427.31 in the discharge codes. Also, ARIC participants were classified as AF cases if the underlying cause of death was AF (ICD-10 code I48 or ICD-9 code 427.3). In this analysis, cases of early onset AF were identified by any of the three mentioned sources (scheduled study electrocardiogram, hospital discharge ICD-code, or death certificate) in absence of any clinical evidence of structural heart disease. Referent subjects in the ARIC study were 45-64 years of age, had no history of AF at the baseline or in follow up, and no history of a myocardial infarction, heart failure or valvular heart disease at baseline; only subjects of European descent were used for analysis.

The Cleveland Clinic Lone AF Genebank Study consists of subjects from the Cleveland Clinic Lone Atrial Fibrillation Genebank. Subjects included in this analysis were at least 18 years of age and less than 66 years of age with a history of recurring or persistent lone AF, $\$ 50 \%$ coronary artery stenosis in the coronary arteries or with normal stress test results, and had normal left ventricular ejection fraction (LVEF) $250 \%$. Subjects were excluded if they had a history of significant valvular disease, significant coronary artery disease, or a prior history of a myocardial infarction, percutaneous coronary intervention, or coronary artery bypass graft, congenital heart disease, hypertrophic cardiomyopathy, aortic dissection or repair, or latest LVEF $<50 \%$. Referent subjects were drawn from the Genebank at the Cleveland Clinic Heart Center genotyped as part of a prior case control GWAS of myocardial infarction. Referent subjects were included if they had no history of AF at any age, and otherwise met the inclusion criteria as described above for the early onset AF case cohort.

\section{Replication Cohorts}

Unique early onset AF cases from AFNET that were not genotyped in the initial genomewide association study were used as a replication cohort. The Vanderbilt Lone AF Registry 29 consists of patients between 18 and 65 years of age with documented AF in the absence of hypertension, heart failure, coronary disease, or significant valve disease by echocardiography. Consecutive patients with $\mathrm{AF}$ were prospectively enrolled beginning in October 2002 from the Vanderbilt Cardiology and Arrhythmia Clinics, the emergency department, and in-patient services.29 Referent subjects were free from AF as documented by electrocardiography. 


\section{Genotyping}

For lone AF, the genotyping platform utilized in each study included: Affymetrix 6.0 (MGH, ARIC), Affymetrix 5.0 (FHS), Illumina 370 CNV (HVH, CHS), Illumina 330 (AFNET, KORA S4), Illumina Hap550 v3 and Illumina Hap610 v1 (Cleveland Clinic Cases), and Perlegen GV4 (Cleveland Clinic Controls). Additional information on the genotyping exclusions in each cohort are provided in Supplemental Table 1. Replication genotyping was performed for rs13376333 and rs13038095 using a TaqMan assay (Applied Biosystems, Inc., Foster City, CA) in the Vanderbilt Lone AF Registry, and iPlex single base primer extension with MALDI-TOF mass spectrometry (Sequenom, San Diego, CA) for AFNET and KORA S4. The AGES and Rotterdam Studies were genotyped on the Illumina CNV370 and Infinium HumanHap550 platforms, respectively.

\section{Statistical Analysis}

For the lone AF meta-analysis, over 2.5 million HapMap SNPs were imputed within each study using the CEU population. Mach v1.0.1× (http://www.sph.umich.edu/csg/abecasis/ MACH/) was used by AFNET, KORA S4, MGH, FHS, ARIC, and Cleveland Clinic; BIMBAM30 was used by HVH and CHS. In studies for which population structure was associated with the lone AF phenotype (FHS/MGH, CHS/HVH, Cleveland Clinic), analyses were adjusted for the principal components of genotype associated with phenotype,31 or were stratified by identity-by-descent cluster group using the method implemented in PLINK.32 The primary GWAS analysis in each center used logistic regression, adjusting for age at DNA draw, sex, and hypertension status. ARIC also adjusted for study site. The $\mathrm{HVH} / \mathrm{CHS}$ analysis was not adjusted for age because of limited overlap in ages between cases and controls. Each SNP was modeled using an additive genetic effect. The ratio of observed to expected variance in the imputed SNP genotype counts was used as a quality control metric for imputed SNPs.33 For each SNP, all studies with variance ratios greater than 0.10 were included in meta-analyses. A fixed effects model was used for meta-analysis of the genotype logistic regression parameters (log odds ratios), using inverse variance weights as implemented in the meta-analysis utility METAL (http:// www.sph.umich.edu/csg/abecasis/metal/index.html). Prior to meta-analysis, genomic control was applied to each study with genomic control inflation factor $(\lambda)>1.0$ by multiplying the standard error of the SNP regression parameter by the square-root of the study-specific $\lambda$. A total of 2,445,595 SNPs with average minor allele frequencies greater than or equal to 0.01 across participating studies were included in meta-analyses. To guard against reporting false positives due to results from a single study, we omitted SNPs with results from fewer than 3 studies available and SNPs with extreme results in a single study $\left(\mathrm{p}<10^{-8}\right)$ with no consistent support at $\mathrm{P}<0.05$ from other studies. We pre-specified a $\mathrm{P}<5 \times 10^{-8}$ corresponding to Bonferroni adjustment for 1 million independent tests 18 as our criterion for genome-wide significance.

We sought to determine if the top SNP at each locus for lone AF that exceeded our threshold for genome-wide significance was also associated with more typical forms of AF observed in the community by performing an in silico replication in the Age, Gene/ Environment Susceptibility Study (AGES) and the Rotterdam Study. 


\section{Supplementary Material}

Refer to Web version on PubMed Central for supplementary material.

\section{Authors}

Patrick T. Ellinor ${ }^{1,2,3,60}$, Kathryn L. Lunetta ${ }^{4,5,60}$, Nicole L. Glazer ${ }^{6,60}$, Arne Pfeufer $^{7,8,60}$, Alvaro Alonso 9,60 , Mina K. Chung ${ }^{10,60}$, Moritz F. Sinner ${ }^{11,60}$, Paul I. W. de Bakker ${ }^{12,13}$, Martina Mueller ${ }^{11,14,15}$, Steven A. Lubitz ${ }^{1,16}$, Ervin Fox ${ }^{17}$, Dawood Darbar ${ }^{18}$, Nicholas L. Smith ${ }^{19,20}$, Jonathan D. Smith ${ }^{21}$, Renate B. Schnabel ${ }^{5}$, Elsayed Z. Soliman ${ }^{22}$, Kenneth M. Rice ${ }^{23}$, David R. Van Wagoner ${ }^{10}$, Britt-M. Beckmann ${ }^{11}$, Charlotte van Noord ${ }^{24,25,26}$, Ke Wang ${ }^{4}$, Georg B. Ehret ${ }^{27}$, Jerome I. Rotter ${ }^{28}$, Stanley L. Hazen ${ }^{29}$, Gerhard Steinbeck ${ }^{11}$, Albert V. Smith ${ }^{30,31}$, Lenore J. Launer ${ }^{32}$, Tamara B. Harris ${ }^{32}$, Seiko Makino ${ }^{1}$, Mari Nelis ${ }^{33,34}$, David J. Milan ${ }^{1,2}$, Siegfried Perz ${ }^{35,36}$, Tõnu Esko ${ }^{33,34,37}$, Anna Köttgen ${ }^{38}$, Susanne Moebus $^{39}$, Christopher Newton-Cheh ${ }^{1,3,13,40}$, Man Li ${ }^{38}$, Stefan Möhlenkamp ${ }^{41}$, Thomas J. Wang ${ }^{5,40}$, W.H. Linda Kao ${ }^{38}$, Ramachandran S. Vasan ${ }^{5,42}$, Markus M. Nöthen ${ }^{43}$, Calum A. MacRae ${ }^{44}$, Bruno H. Ch. Stricker24,26,45,46,47, Albert Hofman ${ }^{24,26}$, André G. Uitterlinden $24,26,45$, Daniel Levy ${ }^{48}$, Eric Boerwinkle ${ }^{49}$, Andres Metspalu ${ }^{33,34,37}$, Eric J. Topol ${ }^{50}$, Aravinda Chakravarti51, Vilmundur Gudnason $^{30,31}$, Bruce M. Psaty ${ }^{52}$, Dan M. Roden ${ }^{18}$, Thomas Meitinger ${ }^{7,8}$, H.-Erich Wichmann 53,54,55, Jacqueline C.M. Witteman24,26,60, John Barnard56,60, Dan E. Arking ${ }^{51,60}$, Emelia J. Benjamin $5,57,58,60$, Susan R. Heckbert ${ }^{59,60}$, and Stefan Kääb 11,60

\section{Affiliations}

${ }^{1}$ Cardiovascular Research Center, Massachusetts General Hospital, Boston, MA ${ }^{2}$ Cardiac Arrhythmia Service, Massachusetts General Hospital, Boston, MA ${ }^{3}$ Center for Human Genetic Research, Massachusetts General Hospital, Boston, MA ${ }^{4}$ Department of Biostatistics, School of Public Health, Boston University, Boston, MA ${ }^{5}$ National Heart, Lung and Blood Institute's Framingham Heart Study, Framingham, MA ${ }^{6}$ Cardiovascular Health Research Unit and Department of Medicine, University of Washington, Seattle, WA ${ }^{7}$ Institute of Human Genetics, Klinikum Rrchts der Isar Technische Universität München, Munich, Germany ${ }^{8}$ Institute of Human Genetics, Helmholtz Zentrum München, Deutsches Forschungszentrum für Gesundheit und Umwelt, Neuherberg, Germany ${ }^{9}$ Division of Epidemiology and Community Health, School of Public Health, University of Minnesota, Minneapolis, MN ${ }^{10}$ Department of Cardiovascular Medicine, Heart and Vascular Institute, Department of Molecular Cardiology, Lerner Research Institute, Cleveland Clinic, Cleveland, $\mathrm{OH}^{11}$ Department of Medicine I, University Hospital Grosshadern, Ludwig-Maximilians-University, Munich, Germany ${ }^{12}$ Division of Genetics, Department of Medicine, Brigham and Women's Hospital, Harvard Medical School, Boston, MA $02115{ }^{13}$ Program in Medical and Population Genetics, Broad Institute of Harvard and MIT, Cambridge, MA ${ }^{14}$ Institute of Epidemiology, Helmholtz Zentrum München, German Research Center for Environmental Health, Neuherberg, Germany ${ }^{15}$ Institute of Medical Informatics, Biometry and 
Epidemiology, Ludwig-Maximilians-Universität, Munich, Germany ${ }^{16}$ Division of Preventive Medicine, Brigham and Women's Hospital, Boston, MA ${ }^{17}$ Department of Medicine, University of Mississippi Medical Center, Jackson, MS ${ }^{18}$ Divisions of Cardiovascular Medicine and Clinical Pharmacology, Vanderbilt University School of Medicine, Nashville, TN ${ }^{19}$ Department of Epidemiology, University of Washington, Seattle, WA ${ }^{20}$ Seattle Epidemiologic Research and Information Center of the Department of Veterans Affairs Office of Research and Development, Seattle, Washington ${ }^{21}$ Department of Cell Biology, Lerner Research Institute, Department of Cardiovascular Medicine, Heart and Vascular Institute, Cleveland Clinic, Cleveland, $\mathrm{OH}^{22}$ Epidemiological Cardiology Research Center (EPICARE), Department of Epidemiology and Prevention, Wake Forest University School of Medicine, Winston Salem, NC ${ }^{23}$ Department of Biostatistics, University of Washington, Seattle, WA ${ }^{24}$ Department of Epidemiology, Erasmus Medical Center, Rotterdam, The Netherlands ${ }^{25}$ Dutch Medicines Evaluation Board, The Hague, The Netherlands ${ }^{26}$ Netherlands Consortium on Healthy Aging (NCHA) Leiden, The Netherlands ${ }^{27}$ McKusick-Nathans Institute of Genetic Medicine, Johns Hopkins University School of Medicine, Baltimore, MD ${ }^{28}$ Medical Genetics Institute, Cedars-Sinai Medical Center, Los Angeles, CA ${ }^{29}$ Department of Cardiovascular Medicine, Heart and Vascular Institute, Cleveland Clinic, Cleveland, $\mathrm{OH}^{30}$ Icelandic Heart Association, Kópavogur, Iceland ${ }^{31}$ University of Iceland, Reykjavik, Iceland ${ }^{32}$ Laboratory of Epidemiology, Demography, and Biometry, Intramural Research Program, National Institute on Aging, Bethesda, MD ${ }^{33}$ Estonian Biocenter, Genotyping Core Facility, Tartu, Estonia ${ }^{34}$ Institute of Molecular and Cell Biology, University of Tartu, Tartu, Estonia ${ }^{35}$ Institute for Biological and Medical Imaging, Helmholtz Zentrum München, Germany ${ }^{36}$ German Research Center for Environmental Health, Neuherberg, Germany ${ }^{37}$ Estonian Genome Center, University of Tartu, Tartu, Estonia ${ }^{38}$ Department of Epidemiology, Johns Hopkins University, Baltimore, MD ${ }^{39}$ Institute of Medical Informatics, Biometry and Epidemiology, University Duisburg-Essen, Essen, Germany ${ }^{40}$ Cardiology Division, Massachusetts General Hospital, Boston, MA ${ }^{41}$ Department of Cardiology, West German Heart Center Essen, University Duisburg-Essen, Essen, Germany ${ }^{42}$ Departments of Medicine, Preventive Medicine and Cardiology sections, Boston University School of Medicine ${ }^{43}$ Institute of Human Genetics, University of Bonn and Department of Genomics, Life \& Brain Center Bonn, D-53127 Bonn ${ }^{44}$ Cardiovascular Division, Brigham and Woman's Hospital, Boston, MA ${ }^{45}$ Department of Internal Medicine, Erasmus Medical Center, Rotterdam, The Netherlands ${ }^{46}$ Inspectorate for Health Care, The Hague, The Netherlands ${ }^{47}$ Department of Medical Informatics, Erasmus Medical Center, Rotterdam, The Netherlands ${ }^{48}$ Center for Population Studies, National Heart, Lung, and Blood Institute, Bethesda, MD ${ }^{49}$ University of Texas Health Science Center at Houston, Human Genetics Center and Institute for Molecular Medicine, Houston, TX ${ }^{50}$ Scripps Translational Science Institute, The Scripps Research Institute, Scripps Health, La Jolla, CA ${ }^{51}$ McKusick-Nathans Institute of Genetic Medicine, Johns Hopkins University School of Medicine, Baltimore, MD ${ }^{52}$ Cardiovascular Health Research Unit, Departments of Medicine, Epidemiology, and Health Services, 
University of Washington, and Group Health Research Institute, Group Health, Seattle, WA ${ }^{53}$ Institute of Epidemiology, Helmholtz Zentrum München, German Research Center for Environmental Health, Neuherberg, Germany ${ }^{54}$ Institute of Medical Informatics, Biometry and Epidemiology, Ludwig-Maximilians-Universität, Munich, Germany ${ }^{55}$ Klinikum Grosshadern, Munich, Germany ${ }^{56}$ Department of Quantitative Health Sciences, Lerner Research Institute, Cleveland Clinic, Cleveland, $\mathrm{OH}^{57}$ Department of Epidemiology, School of Public Health, Boston University, Boston, MA ${ }^{58}$ Cardiology and Preventive Medicine Division, Evans Department of Medicine, Whitaker Cardiovascular Institute, Boston University School of Medicine, Boston, MA ${ }^{59}$ Department of Epidemiology, University of Washington, and Group Health Research Institute, Group Health, Seattle, WA

\section{Abbreviations}

$\begin{array}{ll}\text { AF } & \text { atrial fibrillation } \\ \text { AFNET } & \text { The German Competence Network on Atrial Fibrillation } \\ \text { GWAS } & \text { genome wide association study } \\ \text { ARIC } & \text { Atherosclerosis Risk in Communities Study } \\ \text { CHS } & \text { Cardiovascular Health Study } \\ \text { FHS } & \text { Framingham Heart Study } \\ \text { HVH } & \text { Heart and Vascular Health Study } \\ \text { MGH } & \text { Massachusetts General Hospital Atrial Fibrillation Study } \\ \text { SNP } & \text { single nucleotide polymorphisms }\end{array}$

\section{References}

1. Fuster V, et al. ACC/AHA/ESC 2006 Guidelines for the Management of Patients with Atrial Fibrillation: a report of the American College of Cardiology/American Heart Association Task Force on Practice Guidelines and the European Society of Cardiology Committee for Practice Guidelines (Writing Committee to Revise the 2001 Guidelines for the Management of Patients With Atrial Fibrillation): developed in collaboration with the European Heart Rhythm Association and the Heart Rhythm Society. Circulation. 2006; 114:e257-354. [PubMed: 16908781]

2. Jahangir A, et al. Long-term progression and outcomes with aging in patients with lone atrial fibrillation: a 30-year follow-up study. Circulation. 2007; 115:3050-6. [PubMed: 17548732]

3. Ellinor PT, Yoerger DM, Ruskin JN, MacRae CA. Familial aggregation in lone atrial fibrillation. Hum Genet. 2005; 118:179-84. [PubMed: 16133178]

4. Fox CS, et al. Parental atrial fibrillation as a risk factor for atrial fibrillation in offspring. JAMA. 2004; 291:2851-5. [PubMed: 15199036]

5. Arnar DO, et al. Familial aggregation of atrial fibrillation in Iceland. Eur Heart J. 2006; 27:708-12. [PubMed: 16428254]

6. Gudbjartsson DF, et al. Variants conferring risk of atrial fibrillation on chromosome 4q25. Nature. 2007; 448:353-7. [PubMed: 17603472]

7. Benjamin EJ, et al. Variants in ZFHX3 are associated with atrial fibrillation in individuals of European ancestry. Nat Genet. 2009; 41:879-81. [PubMed: 19597492] 
8. Gudbjartsson DF, et al. A sequence variant in ZFHX3 on 16q22 associates with atrial fibrillation and ischemic stroke. Nat Genet. 2009; 41:876-8. [PubMed: 19597491]

9. Kozlowski D, et al. Lone Atrial Fibrillation - What Do We Know? Heart. 2009

10. Kohler M, et al. Small-conductance, calcium-activated potassium channels from mammalian brain. Science. 1996; 273:1709-14. [PubMed: 8781233]

11. $\mathrm{Xu} \mathrm{Y}$, et al. Molecular identification and functional roles of a $\mathrm{Ca}(2+)$-activated $\mathrm{K}+$ channel in human and mouse hearts. J Biol Chem. 2003; 278:49085-94. [PubMed: 13679367]

12. Tuteja D, et al. Differential expression of small-conductance Ca2+-activated K+ channels SK1, SK2, and SK3 in mouse atrial and ventricular myocytes. Am J Physiol Heart Circ Physiol. 2005; 289:H2714-23. [PubMed: 16055520]

13. Ozgen N, et al. Early electrical remodeling in rabbit pulmonary vein results from trafficking of intracellular SK2 channels to membrane sites. Cardiovasc Res. 2007; 75:758-69. [PubMed: 17588552]

14. Nattel S. New ideas about atrial fibrillation 50 years on. Nature. 2002; 415:219-26. [PubMed: 11805846]

15. Zhang Q, et al. Functional roles of a Ca2+-activated $\mathrm{K}+$ channel in atrioventricular nodes. Circ Res. 2008; 102:465-71. [PubMed: 18096820]

16. Monaghan AS, et al. The SK3 subunit of small conductance $\mathrm{Ca} 2+-$ activated $\mathrm{K}+$ channels interacts with both SK1 and SK2 subunits in a heterologous expression system. J Biol Chem. 2004; 279:1003-9. [PubMed: 14559917]

17. Taylor MS, et al. Altered expression of small-conductance Ca2+-activated K+ (SK3) channels modulates arterial tone and blood pressure. Circ Res. 2003; 93:124-31. [PubMed: 12805243]

18. The International HapMap Project. Nature. 2003; 426:789-96. [PubMed: 14685227]

19. Newton-Cheh C, et al. Genome-wide association study identifies eight loci associated with blood pressure. Nat Genet. 2009

20. Johnson AD, et al. SNAP: a web-based tool for identification and annotation of proxy SNPs using HapMap. Bioinformatics. 2008; 24:2938-9. [PubMed: 18974171]

21. Kaab S, et al. Large scale replication and meta-analysis of variants on chromosome 4q25 associated with atrial fibrillation. Eur Heart J. 2009

22. Wichmann HE, Gieger C, Illig T. KORA-gen--resource for population genetics, controls and a broad spectrum of disease phenotypes. Gesundheitswesen. 2005; 67(Suppl 1):S26-30. [PubMed: 16032514]

23. Dawber TR, Meadors GF, Moore FE Jr. Epidemiological approaches to heart disease: the Framingham Study. Am J Public Health Nations Health. 1951; 41:279-81. [PubMed: 14819398]

24. Kannel WB, Feinleib M, McNamara PM, Garrison RJ, Castelli WP. An investigation of coronary heart disease in families. The Framingham offspring study. Am J Epidemiol. 1979; 110:281-90. [PubMed: 474565]

25. Splansky GL, et al. The Third Generation Cohort of the National Heart, Lung, and Blood Institute's Framingham Heart Study: design, recruitment, and initial examination. Am J Epidemiol. 2007; 165:1328-35. [PubMed: 17372189]

26. Heckbert SR, Li G, Cummings SR, Smith NL, Psaty BM. Use of alendronate and risk of incident atrial fibrillation in women. Arch Intern Med. 2008; 168:826-31. [PubMed: 18443257]

27. Fried LP, et al. The Cardiovascular Health Study: design and rationale. Ann Epidemiol. 1991; 1:263-76. [PubMed: 1669507]

28. The Atherosclerosis Risk in Communities (ARIC) Study: design and objectives. The ARIC investigators. Am J Epidemiol. 1989; 129:687-702. [PubMed: 2646917]

29. Darbar D, Motsinger AA, Ritchie MD, Gainer JV, Roden DM. Polymorphism modulates symptomatic response to antiarrhythmic drug therapy in patients with lone atrial fibrillation. Heart Rhythm. 2007; 4:743-9. [PubMed: 17556195]

30. Servin B, Stephens M. Imputation-based analysis of association studies: candidate regions and quantitative traits. PLoS Genet. 2007; 3:e114. [PubMed: 17676998]

31. Price AL, et al. Principal components analysis corrects for stratification in genome-wide association studies. Nat Genet. 2006; 38:904-9. [PubMed: 16862161] 
32. Purcell S, et al. PLINK: a tool set for whole-genome association and population-based linkage analyses. Am J Hum Genet. 2007; 81:559-75. [PubMed: 17701901]

33. de Bakker PI, et al. Practical aspects of imputation-driven meta-analysis of genome-wide association studies. Hum Mol Genet. 2008; 17:R122-8. [PubMed: 18852200] 


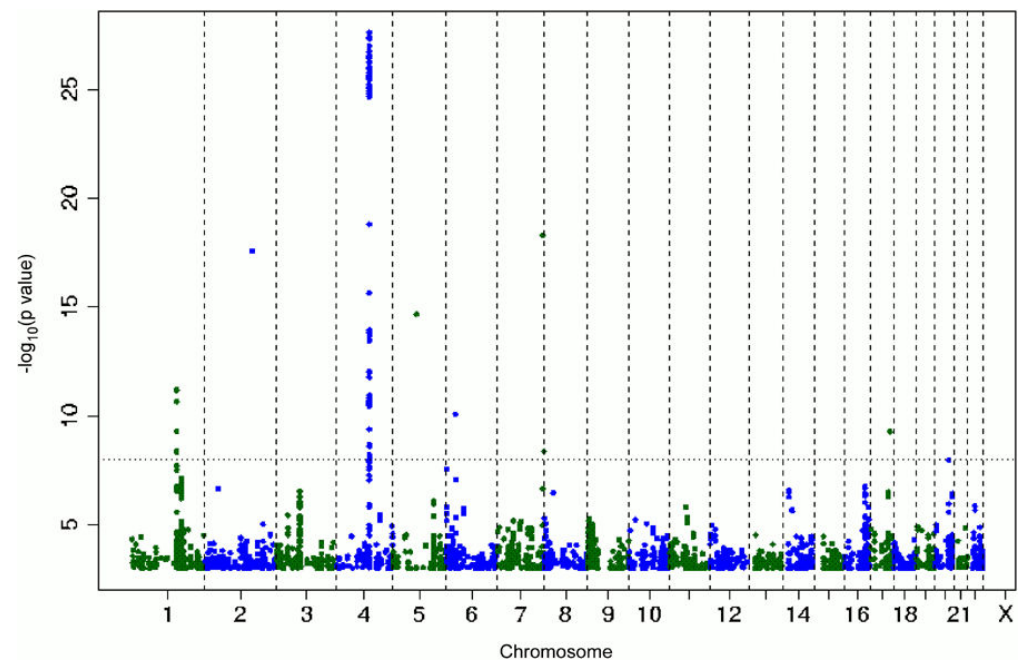

Figure 1. Manhattan plot of meta-analysis results for genome-wide association of lone AF The $-\log _{10}$ (p value) is plotted against the physical positions of each SNP on each chromosome. The threshold for genome-wide significance, $P<5 \times 10^{-8}$, is indicated by the dashed line. 


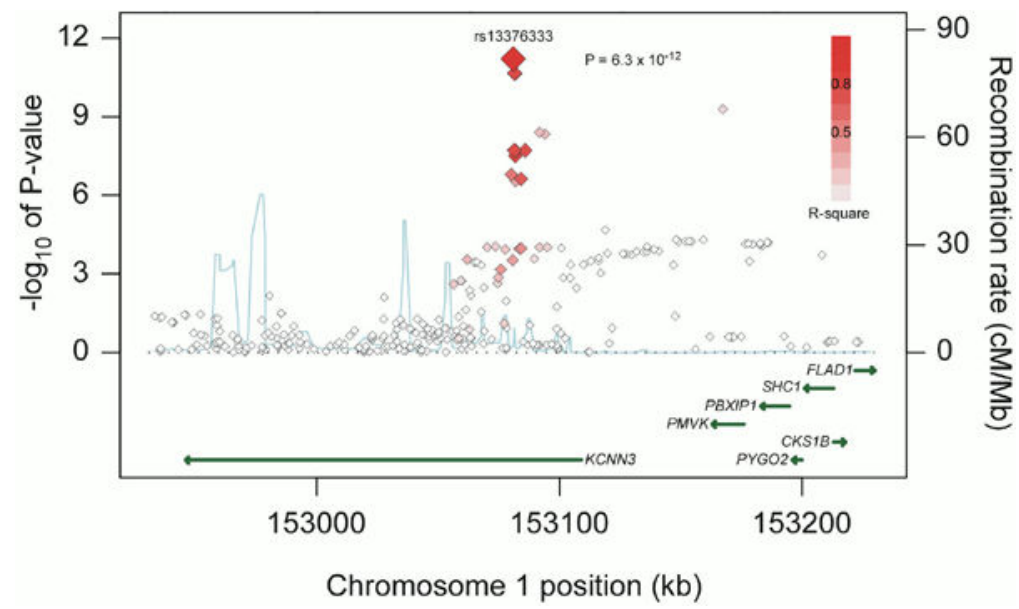

Figure 2. Regional plot for locus on chromosome 1 associated with lone atrial fibrillation Figures prepared using SNAP20. SNPs are plotted with the meta-analysis $P$-value and genomic position (NCBI Build 36). The SNP of interest is labeled. The strength of the linkage disequilibrium (LD) is indicated by gradient of red. Estimated recombination rates are shown by the blue line and gene annotations are indicated by dark green arrows. LD and recombination rates are based on the CEU HapMap release 22. 


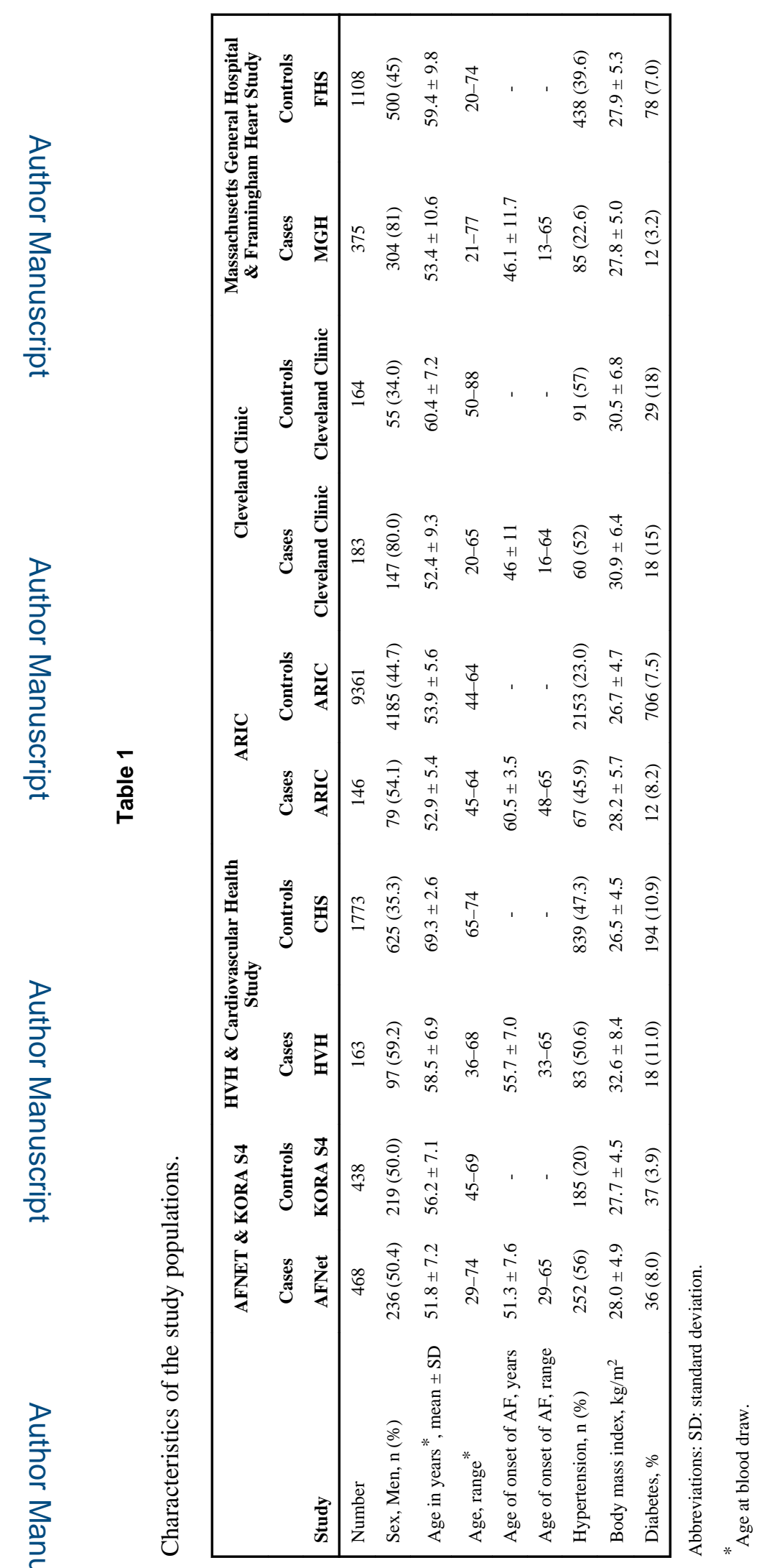

Nat Genet. Author manuscript; available in PMC 2010 September 01. 


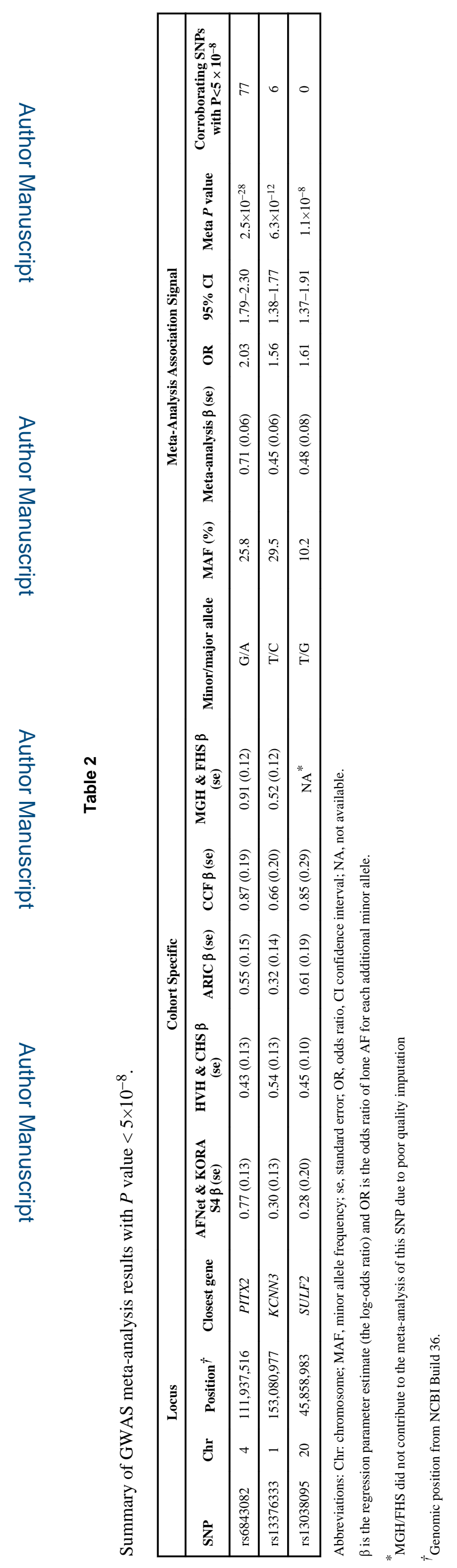




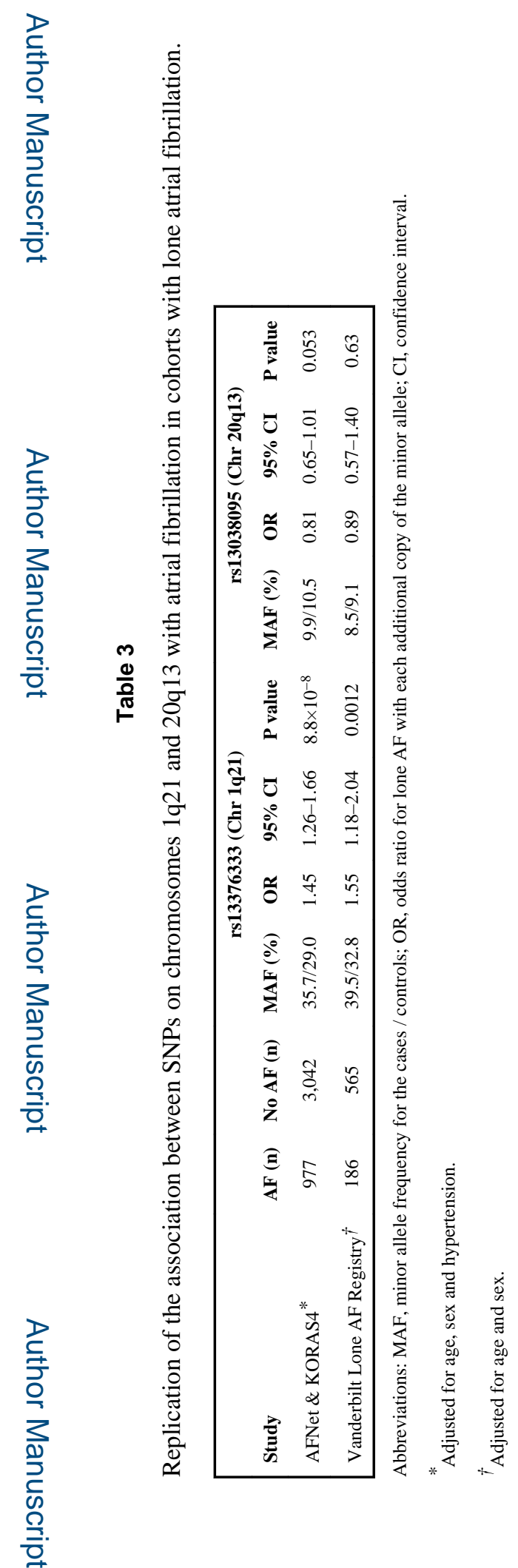

Nat Genet. Author manuscript; available in PMC 2010 September 01. 